\title{
Các nhân tố ảnh hưởng đến sự hài lòng của khách hàng cá nhân về dịch vụ Internet Banking của các ngân hàng thương mại tại Thành phố Hồ Chí Minh
}

\section{Determinants affecting individual customer satisfaction about Internet Banking services of commercial banks in Ho Chi Minh City}

\author{
Nguyễn Thị Bình Minh ${ }^{1 *}$, Khúc Đình Nam ${ }^{1}$, Phan Thị Lệ Hằng ${ }^{1}$ \\ ${ }^{1}$ Trường Đại học Nông Lâm Thành phố Hồ Chí Minh, Việt Nam \\ "Tác giả liên hệ, Email: binhminh_sunshine@hcmuaf.edu.vn
}

THÔNG TIN

TÓM TĂT

DOI: $10.46223 /$ HCMCOUJS. econ.vi.16.3.1299.2021

Ngày nhận: 02/11/2020

Ngày nhận lại: 17/12/2020

Duyệt đăng: 28/12/2020

Tù khóa:

internet banking; khách hàng cá nhân; sự hài lòng

Keywords:

internet banking services; individual customers; satisfaction
Nghiên cứu này tập trung xác định các nhân tố ảnh hưởng đến sự hài lòng của khách hàng cá nhân về dịch vụ Internet Banking của các ngân hàng thương mại tại Thành phố Hồ Chí Minh. Mô hình nghiên cứu chủ yếu dựa trên mô hình sự thành công của hệ thống thông tin của Delone và Mclean (2003). Dữ liệu nghiên cứu thực nghiệm được thu thập thông qua việc khảo sát 385 khách hàng cá nhân đang sử dụng dịch vụ Internet Banking tại địa bàn nghiên cứu. Thông qua việc phân tích nhân tố khám phá EFA, phân tích nhân tố khẳng định CFA, phân tích mô hình cấu trúc tuyến tính $\mathrm{SEM}$ và kiểm định ước lượng mô hình bằng Bootstrap, nhóm tác giả đã khám phá ra rằng sự tin tưởng và chất lượng dịch vụ tác động tích cực đến sự hài lòng, trong đó sự tin tưởng chịu ảnh hưởng của 03 nhân tố là chất lượng thông tin, chất lượng hệ thống và chất lượng dịch vụ. Từ kết quả trên, nhóm tác giả trình bày một số khuyến nghị để giúp cho các ngân hàng thương mại gia tăng sự hài lòng của khách hàng khi sử dụng dịch vụ Internet Banking của mình.

ABSTRACT
This research objective is to identify factors affecting
individual customer satisfaction with Internet Banking services of
commercial banks in Ho Chi Minh City. The research model was
developed based on the Information System Success Model (ISS)
of Delone and Mclean (2003). The empirical data was collected
through 385 questionnaires surveyed by individual customers
using Internet Banking services in the research area. By applying
the exploratory factor analysis EFA, confirmatory factor analysis
CFA, structural equation modeling SEM, and Bootstrap model
estimation test, the authors have discovered that trust and service
quality positively affected satisfaction. Trust was influenced by
03 factors of system quality, information quality, and service
quality. From the above research results, the authors also showed
some recommendations to help commercial banks increase
customer satisfaction when using their Internet Banking services.




\section{Mở đầu}

Trong thời đại 4.0, Internet ngày càng trở nên thông dụng và trên toàn cầu. Đầu năm 2020, số lượng người đang sử dụng internet là hơn 4.5 tỷ người trên toàn thế giới (theo Báo cáo về Digital toàn cầu của We Are Social và Hootsuite, 2020. Đặc biệt, tại Việt Nam, số lượng thuê bao di động là 145.8 triệu chiếm $150 \%$ so với dân số Việt Nam phát triển mạnh mẽ và số lượng người dùng Internet là 68.17 triệu người. Tỷ lệ người Việt Nam sở hữu thiết bị di động là $94 \%$, laptop/máy tính bàn là $65 \%$, máy tính bảng là $32 \%$. Với tỷ lệ sử dụng các thiết bị nhiều như vậy, 03 hoạt động người dân dành nhiều thời gian nhất trong một ngày là: sử dụng Internet, sử dụng mạng xã hội và xem truyền hình. Điều này cho thấy sự thâm nhập của Internet vào cuộc sống hàng ngày của người dân Việt Nam hiện nay là vô cùng lớn.

Trong thời gian qua, một trong những mục tiêu lớn của Chính phủ và Ngân hàng Nhà nước Việt Nam (Đề án Phát triển thanh toán không dùng tiền mặt tại Việt Nam giai đoạn 2016 - 2020) quan tâm phát triển là không dùng tiền mặt trong quá trình thanh toán và đã gặt hái được những thành công nhất định. Theo số liệu thống kê năm 2018 của Ngân hàng Thế giới (as cited in VA, 2019), số lượng giao dịch không sử dụng tiền mặt tại Việt Nam đạt mức 4.9\% mức độ thấp nhất khi so sánh với một số nước trong khu vực, cụ thể $59.7 \%$ tại Thái Lan, 89\% tại Malaysia, và 26.1\% tại Trung Quốc (Le, 2019). Hiệp hội ngân hàng Việt Nam đã công bố sự gia tăng rất mạnh về số lượng giao dịch tài chính qua điện thoại di động và Internet trong 03 tháng đầu năm 2019 so với cùng kỳ năm trước, cụ thể: đối với kênh Internet đã tăng $68.8 \%$ về số lượng và $13.4 \%$ về giá trị; đối với kênh điện thoại di động đã tăng $97.7 \%$ về số lượng và $232.3 \%$ về giá trị (Hoang Liem, 2019). Theo số liệu thống kê của Vụ thanh toán Ngân hàng Nhà nước Việt Nam (as cited in VA, 2019), số lượng người dân Việt Nam trên 15 tuổi đã có tài khoản ngân hàng đạt trên hơn 40 triệu người; số lượng tổ chức đã cung cấp dịch vụ thanh toán qua Internet là 78; số lượng tổ chức cung cấp dịch vụ thanh toán qua điện thoại di động là 44; tổng giá trị giao dịch qua Internet Banking và Mobile Banking trong quý II năm 2019 lần lượt là 9,500 nghìn tỷ đồng và 1,760 nghìn tỷ đồng (VA, 2019).

Hiện nay, có rất nhiều nghiên cứu về Internet Banking, được chia làm 03 hướng nghiên cứu chính: một là về các yếu tố ảnh hưởng đến ý định sử dụng dịch vụ Internet Banking; hai là về sự hài lòng của khách hàng về chất lượng dịch vụ Internet Banking; ba là về các động lực tại sao các ngân hàng phát triển dịch vụ Internet Banking (Do, 2016). Hướng nghiên cứu về sự hài lòng về chất lượng dịch vụ Internet Banking của khách hàng đã thu hút nhiều sự quan tâm của nhiều tác giả chẳng hạn như Y. I. Hsu và Nguyen (2016), Musiime và Ramadhan (2011), Nimako, Gyamfi, và Wandaogou (2013), Seyal và Rahim (2011), Tran va Nguyen (2011), ... Những nghiên cứu này đều dựa trên một số mô hình lý thuyết quen thuộc về chất lượng dịch vụ như E-SERQUAL, E-SQ (E- Service Quality) và SERQUAL.

Tuy nhiên, Internet Banking là một dịch vụ dựa trên ứng dụng của hệ thống công nghệ thông tin, do đó để phát triển dịch vụ Internet Banking thành công cũng như tối đa hóa sự hài lòng của khách hàng, theo mô hình Information System Success (ISS) của Delone và Mclean (2003) ngoài chất lượng dịch vụ còn có các nhân tố khác là chất lượng hệ thống và chất lượng thông tin. Bên cạnh đó, sự hài lòng của khách hàng còn chịu sự tác động bởi sự tin tưởng (Lee \& Chung, 2009). Sự ảnh hưởng của ba nhân tố mới này chưa được các nhà nghiên cứu tại Việt Nam đưa vào phân tích. Do đó, nhóm tác giả đã tiến hành nghiên cứu này để giải quyết khoảng trống nghiên cứu về dịch vụ Internet Banking tại Việt Nam.

Mục tiêu của nghiên cứu này là xác định các nhân tố ảnh hưởng đến sự hài lòng của khách hàng về dịch vụ Internet Banking chủ yếu dựa trên mô hình ISS. Trên cơ sở đó, bài báo 
trình bày một số đề xuất cho các ngân hàng nhằm thỏa mãn tối đa sự hài lòng của khách hàng về Internet Banking. Nghiên cứu đã tiến hành điều tra 385 khách hàng cá nhân tại Thành phố Hồ Chí Minh đã sử dụng dịch vụ Internet Banking. Kết cấu của nghiên cứu bao gồm: phần một mở đầu, phần hai trình bày cơ sở lý thuyết và mô hình nghiên cứu, phần ba phương pháp nghiên cứu, phần bốn trình bày kết quả nghiên cứu, phần cuối cùng là kết luận và khuyến nghị.

\section{Cơ sở lý thuyết và mô hình nghiên cứu}

\subsection{Lý thuyết nền}

\subsubsection{Internet Banking}

Theo Thông tư số 35/2016/TT-NHNN, dịch vụ Internet Banking là các dịch vụ ngân hàng và dịch vụ trung gian thanh toán được các đơn vị cung cấp thông qua mạng Internet. Khách hàng của dịch vụ Internet Banking bao gồm các tổ chức và cá nhân sử dụng dịch vụ Internet Banking. Hệ thống Internet Banking là một tập hợp có cấu trúc các trang thiết bị phần cứng, phần mềm, cơ sở dữ liệu, hệ thống mạng truyền thông và an ninh bảo mật để sản xuất, truyền nhận, thu thập, xử lý, lưu trữ và trao đổi thông tin số phục vụ cho việc quản lý và cung cấp dịch vụ Internet Banking.

Internet Banking có ưu thế hơn so với hình thức ngân hàng truyền thống. Internet Banking giúp ngân hàng tiết kiệm chi phí hoạt động, gia tăng hiệu quả hoạt động kinh doanh, nhanh chóng thâm nhập thị trường mới, cũng như giúp ngân hàng nâng cao uy tín và khả năng cạnh tranh. Mặt khác, Internet Banking còn cung cấp cho khách hàng những tiện ích mới như tiết chi phí và thời gian vì khách hàng có thể thực hiện giao dịch bất cứ thời điểm và thời gian nào một cách hiệu quả và nhanh chóng mà không phụ thuộc vào giờ giao dịch của ngân hàng. Do đó, Internet Banking là lựa chọn tất yếu của các ngân hàng thương mại ở Việt Nam nói riêng và trên thế giới nói chung (Vuong \& Nguyen, 2016).

\subsubsection{Sụ hài lòng của khách hàng}

Sự hài lòng của khách hàng là tổng thể cảm giác và thái độ của khách hàng đối với một số yếu tố ảnh hưởng đến tình hình sử dụng (Bailey \& Pearson, 1983). Đối với những khách hàng hài lòng với dịch vụ được cung cấp, họ thường có xu hướng sử dụng dịch vụ cao hơn, có ý định mua lại mạnh mẽ hơn và thường muốn giới thiệu dịch vụ cho những người khác (Ghane, Fathian, \& Gholamian, 2011). Theo Delone và Mclean (2003), sự hài lòng của khách hàng về dịch vụ mà họ sử dụng được đo lường bằng chất lượng thông tin, chất lượng hệ thống và chất lượng dịch vụ của dịch vụ đó. Ngoài ra, Lee và Chung (2009) đã chứng minh rằng sự hài lòng của khách hàng phụ thuộc vào sự tin tưởng của họ vào dịch vụ.

\subsubsection{Mô hình sự thành công của hệ thống thông tin ISS của Delone và Mclean}

Mô hình sự thành công của hệ thống thông tin ISS được xây dựng lần đầu tiên vào năm 1992 bởi Delone và Mclean. Sau đó, vào năm 2003, hai tác giả này tiếp tục phát triển mô hình ISS mới dựa trên mô hình cũ với mục đích xác định các nhân tố tạo nên sự thành công của hệ thống thông tin thông qua Internet, bao gồm 06 nhân tố là chất lượng thông tin, chất lượng hệ thống, chất lượng dịch vụ, sự hài lòng của người dùng, ý định sử dụng và lợi ích ròng (hay việc nhận thức của khách hàng về vai trò của hệ thống thông tin đối với thành công của tổ chức/công ty). Mô hình ISS mới này đo lường đa chiều và đa biến, cụ thể là chất lượng hệ thống và chất lượng thông tin ảnh hưởng đến sự hài lòng và ý định sử dụng, đồng thời có mối quan hệ tương quan giữa ý định sử dụng và sự hài lòng. Chất lượng hệ thống được hiểu là sự cảm nhận trong quá trình khách hàng sử dụng dịch vụ về tính hữu ích và tính ổn định của hệ thống. Chất lượng thông tin là niềm tin của khách hàng vào tính chính xác, kịp thời và có ích của hệ thống thông tin đối với người dùng. Chất lượng dịch vụ trong mô hình này là việc hướng dẫn khách hàng, bảo trì 
hệ thống và xử lý sự cố phát sinh mà nhà cung cấp hệ thống thông tin sẽ thực hiện trong quá trình cung cấp dịch vụ của mình. Chất lượng dịch vụ có tác động đến sự hài lòng của khách hàng cũng như tác động đến ý định của họ đối với việc sử dụng dịch vụ. Bên cạnh đó, khách hàng sẽ hài lòng khi họ nhận thấy chi phí phải bỏ ra ít hơn so với lợi ích mà họ nhận được trong quá trình sử dụng dịch vụ.

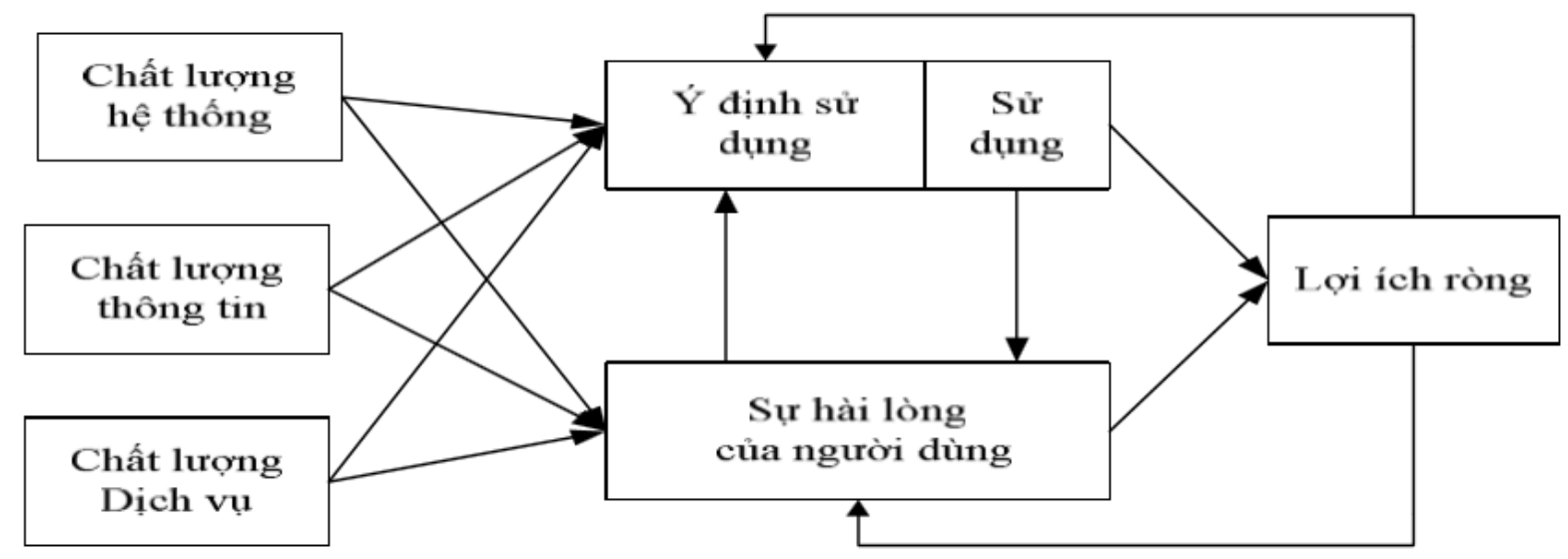

Hình 1. Mô hình ISS của Delone và Mclean (2003)

\subsection{Mô hình và giả thuyết nghiên cứu}

- Chất lượng thông tin: Delone và Mclean (2003), Koo, Wati, và Chung (2013) đã khẳng định chất lượng thông tin là một nhân tố quan trọng ảnh hưởng tích cực đến sự hài lòng về dịch vụ Internet Banking của khách hàng. Lee và Chung (2009) đã chứng minh rằng chất lượng thông tin ảnh hưởng tích cực đến sự hài lòng và sự tin tưởng của khách hàng đối với dịch vụ ngân hàng điện tử.

Hla: Chất lương thông tin có mối quan hệ cùng chiều với sụ hài lòng của khách hàng về dịch vu Internet Banking

H1b: Chất lượng thông tin có mối quan hệ cùng chiều với sụ tin tưởng của khách hàng về dịch vụ Internet Banking

- Chất lượng hệ thống: Theo Delone và Mclean (1992, 2003), C. Liu và Arnett (2000), Lee và Chung (2009) đã khẳng định rằng chất lượng hệ thống là một nhân tố quan trọng ảnh hưởng mạnh đến sự hài lòng và sự tin tưởng của khách hàng. Theo các tác giả, chất lượng hệ thống được đo lường bởi 04 nhân tố chính là dễ sử dụng (Doll \& Torkzadeh, 1988; Gable, Sedera, \& Chan, 2008; Iivari, 2005; Sedera, Gable, \& Chan, 2004), tốc độ giao dịch (Aladwani \& Palvia, 2002; Iivari, 2005; Liao \& Cheung, 2002), bảo mật an ninh (H. S. Hsu, 2008; Madu \& Madu, 2002) và thiết kế giao diện (X. Liu, He, Gao, \& Xie, 2008; Zviran, Glezer, \& Avni, 2006).

H2a: Chất lượng hệ thống có mối quan hệ cùng chiều với sụ hài lòng của khách hàng về dịch vu Internet Banking

H2b: Chất lương hệ thống có mối quan hệ cùng chiều với sụ tin tưởng của khách hàng về dịch vu Internet Banking

- Chất lượng dịch vụ: Delone và Mclean (2003) đã chỉ ra trong mô hình ISS, chất lượng dịch vụ liên quan đến khía cạnh chất lượng dịch vụ hỗ trợ khách hàng và là một nhân tố quan trọng ảnh hưởng tích cực đến sự hài lòng của khách hàng. Bên cạnh đó, Asfour và Haddad (2014), Widiatmika và Subawa (2017), và Chang, Chang, và Chang (2013), đã chứng minh rằng chất lượng dịch vụ tác động tích cực đến sự tin tưởng và hài lòng của khách hàng vào Internet Banking. 
H3a: Chất lương dịch vụ có mối quan hệ cùng chiều với sụ hài lòng của khách hàng về dịch vu Internet Banking

H3b: Chất lương dịch vụ có mối quan hệ cùng chiều với sụ tin tưởng của khách hàng về dịch vu Internet Banking

- Sự tin tưởng: Morgan và Hunt (1994) cho rằng sự tin tưởng nảy sinh khi khách hàng tin tưởng về sự chính trực cũng như sự tin cậy của các nhà cung cấp. Trong kinh doanh, sự tin tưởng được xem là chất xúc tác trong nhiều giao dịch giữa người bán và người mua để gia tăng sự hài lòng của khách hàng có thể đạt được như mong đợi (Yousafzai, Pallister, \& Foxall, 2003). Do đó, khái niệm sự tin tưởng trong nghiên cứu này là niềm tin của khách hàng vào đơn vị tổ chức giao dịch Internet Banking, cũng như niềm tin của khách hàng vào cơ chế tổ chức thực hiện các giao dịch. Theo Mukherjee và Nath (2003), sự tin tưởng có thể được đo lường bằng định hướng công nghệ, danh tiếng và rủi ro nhận thức được. Bên cạnh đó, Lee và Chung (2009) đã khẳng định sự tin tưởng là một nhân tố ảnh hưởng tích cực đến sự hài lòng của khách hàng, khách hàng càng tin tưởng vào dịch vụ ngân hàng trực tuyến thì họ càng hài lòng.

\section{H4: Sự tin tưởng có mối quan hệ cùng chiều với sụ hài lòng của khách hàng về dịch vụ Internet Banking}

Nhóm tác giả đã đề xuất mô hình nghiên cứu của mình dựa trên việc tổng hợp các công trình nghiên cứu đã được công bố có liên quan kết hợp với sự nghiên cứu khám phá trên nền tảng của mô hình ISS của Delone và Mclean (2003). Mô hình nghiên cứu đề xuất về các yếu tố tác động đến sự hài lòng của khách hàng cá nhân về dịch vụ Internet Banking tại Thành phố Hồ Chí Minh với 04 nhóm yếu tố bao gồm: Chất lượng thông tin, Chất lượng hệ thống, Chất lượng dịch vụ và Sự tin tưởng. Mô hình nghiên cứu được trình bày theo hình dưới đây:

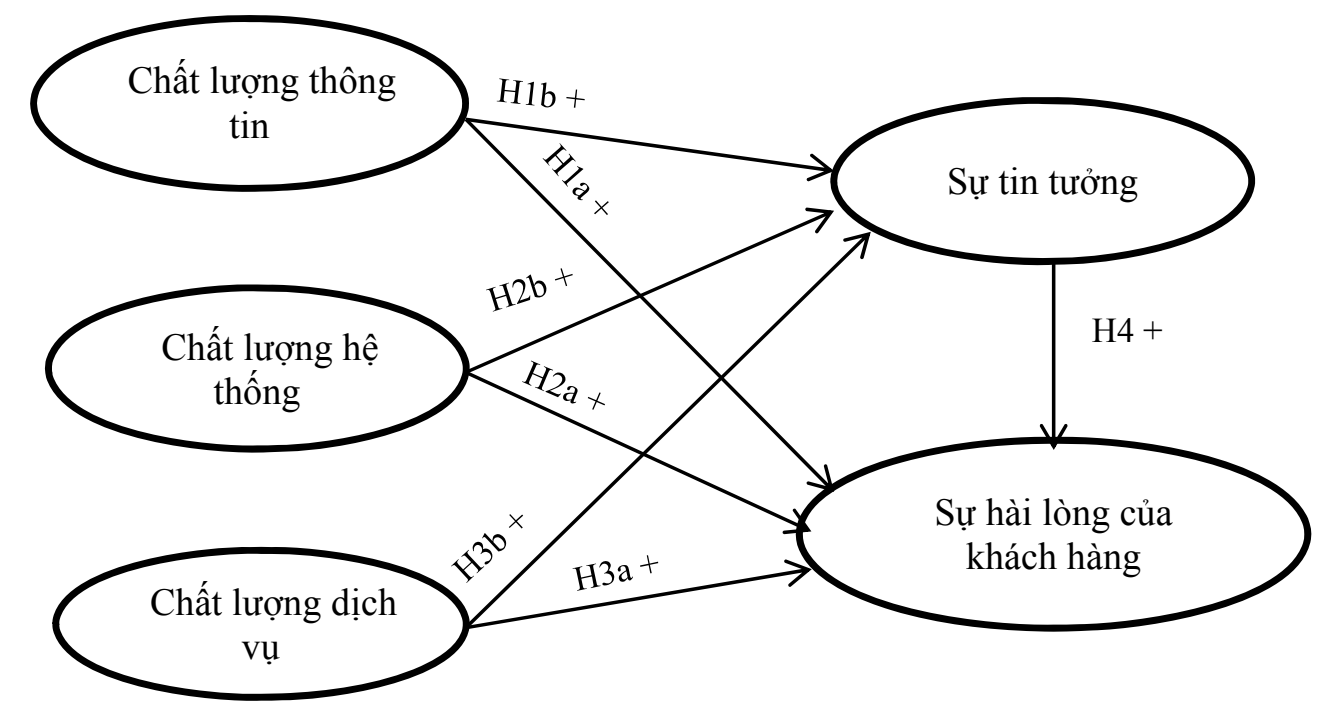

Hình 2. Mô hình nghiên cứu đề xuất

\section{Phương pháp nghiên cứu}

Nghiên cứu trải qua hai giai đoạn: Giai đoạn thứ nhất là nghiên cứu định tính nhằm mục đích xây dựng và củng cố mô hình nghiên cứu lý thuyết thông qua trao đổi trực tiếp với một số chuyên gia trong lĩnh vực ngân hàng cũng như nghiên cứu về các đề tài cùng lĩnh vực nghiên cứu đã được công bố trước đó. Giai đoạn thứ hai là nghiên cứu định lượng để xác định và đo lường các yếu tố tác động đến sự hài lòng của khách hàng cá nhân đối với dịch vụ Internet Banking. Tất cả các thang đo trong nghiên cứu này đều là thang đo đa biến. Các thang đo này sử dụng dạng Likert 5 điểm với: 1 là hoàn toàn không đồng ý, cho đến 5 là hoàn toàn đồng ý. Thang đo trong nghiên cứu được trình bày chi tiết trong bảng 1 dưới đây. 


\section{Bảng 1}

Thang đo

\begin{tabular}{|c|c|c|}
\hline Các nhân tố & Mã hóa & Biến quan sát \\
\hline \multirow{7}{*}{$\begin{array}{l}\text { Chất lượng } \\
\text { thông tin }\end{array}$} & CLTT1 & Nội dung thông tin được hiển thị rất là hữu ích \\
\hline & CLTT2 & Internet Banking luôn cung cấp thông tin đầy đủ \\
\hline & CLTT3 & Các thông tin được cung cấp có liên quan tới việc thực hiện giao dịch \\
\hline & CLTT4 & Thông tin luôn được cập nhật \\
\hline & CLTT5 & Nội dung thông tin luôn được cung cấp nhanh chóng \\
\hline & CLTT6 & Nội dung thông tin được định dạng đẹp mắt và dễ hiểu \\
\hline & CLTT7 & Nội dung thông tin luôn được cung chính xác \\
\hline \multirow{7}{*}{$\begin{array}{l}\text { Chất lượng hệ } \\
\text { thống }\end{array}$} & CLHT1 & Website của Internet Banking dễ sử dụng \\
\hline & CLHT2 & Thủ tục đăng ký Internet Banking dễ dàng, không phức tạp \\
\hline & CLHT3 & Tốc độ kết nối tới Website của ngân hàng nhanh \\
\hline & CLHT4 & Tốc độ xử lý giao dịch của Website nhanh \\
\hline & CLHT5 & $\begin{array}{l}\text { Bảo mật an ninh của Internet Banking đủ tốt để tôi tin tưởng thực } \\
\text { hiện giao dịch }\end{array}$ \\
\hline & CLHT6 & Giao diệnWebsite dễ sử dụng \\
\hline & CLHT7 & Giao diện Website gọn gàng và dễ nhìn \\
\hline \multirow{5}{*}{$\begin{array}{l}\text { Chất lượng } \\
\text { dịch vụ }\end{array}$} & CLDV1 & $\begin{array}{l}\text { Internet Banking có đội ngũ nhân viên luôn thường trực để hỗ trợ } \\
\text { khách hàng }\end{array}$ \\
\hline & CLDV2 & $\begin{array}{l}\text { Nhân viên của Internet Banking luôn tận tâm hướng dẫn và hỗ trợ } \\
\text { khách hàng }\end{array}$ \\
\hline & CLDV3 & Internet Banking cung cấp dịch vụ chính xác và an toàn \\
\hline & CLDV4 & $\begin{array}{l}\text { Những thắc mắc hay khiếu nại của khách hàng luôn được giải } \\
\text { quyết thỏa đáng }\end{array}$ \\
\hline & CLDV5 & Phí dịch vụ của Internet Banking ổn định và cạnh tranh \\
\hline \multirow{4}{*}{ Sự tin tưởng } & NT1 & $\begin{array}{l}\text { Tôi cảm thấy Internet Banking luôn quan tâm đến lợi ích của } \\
\text { khách hàng }\end{array}$ \\
\hline & NT2 & Tôi cảm thấy Internet Banking cung cấp cho tôi dịch vụ tốt \\
\hline & NT3 & $\begin{array}{l}\text { Tôi cảm thấy tin tưởng khi cung cấp các thông tin cá nhân trong } \\
\text { quá trình giao dịch trên Internet Banking }\end{array}$ \\
\hline & NT4 & Nhìn chung, tôi tin tưởng Internet Banking \\
\hline \multirow{5}{*}{ Sự hài lòng } & HL1 & Tôi hài lòng với sự xử lý giao dịch của Internet Banking. \\
\hline & HL2 & $\begin{array}{l}\text { Tôi hài lòng với những dịch vụ được cung cấp trên Internet } \\
\text { Banking }\end{array}$ \\
\hline & HL3 & $\begin{array}{l}\text { Tôi nghĩ rằng tôi đã quyết định đúng đắn khi sử dụng Internet } \\
\text { Banking }\end{array}$ \\
\hline & HL4 & Internet banking cung cấp dịch vụ đúng như sự kỳ vọng của tôi \\
\hline & HL5 & Nhìn chung, tôi hài lòng về Internet Banking \\
\hline
\end{tabular}

Nguồn: Dữ liệu phân tích của nhóm tác giả 
Dữ liệu nghiên cứu được khảo sát trực tiếp khách hàng đã sử dụng Internet Banking hiện đang sống và làm việc tại Thành phố Hồ Chí Minh, thông qua bảng câu hỏi chi tiết theo phương pháp thuận tiện. Kích thước của mẫu áp dụng trong nghiên cứu dựa trên yêu cầu của phân tích nhân tố khám phá EFA và mô hình cấu trúc tuyến tính SEM. Theo nghiên cứu của Hair, Black, Babin, Anderson, và Tatham (1998), kích thước mẫu áp dụng để phân tích EFA phải ít nhất phải bằng 05 lần số biến quan sát. Với mô hình nghiên cứu có 28 biến quan sát, kích thước mẫu tối thiểu là 140 mẫu. Mặt khác, kích thước mẫu cần phải được xem xét trong sự tương quan với số lượng các thông số ước lượng và nếu sử dụng phương pháp ước lượng ML (Maximum Likelihood) thì kích thước mẫu tối thiểu phải từ 100 đến 150 và cỡ mẫu là 250 hoặc lớn hơn để đảm bảo cho tính ổn định và nhất quán của các chỉ số như TLI, CFI (Hair, Anderson, Babin, \& Black, 2010). Theo Cochran (1977) đã chỉ ra công thức tính cỡ mẫu như sau: $n=z^{2}[p(1-p)] / \mathrm{e}^{2}$, trong đó: $n$ là số mẫu cần phỏng vấn, $p$ là ước tính tỷ lệ $\%$ của tổng thế ( $p=50 \%$ là tỷ lệ tối đa), $\mathrm{z}$ là giá trị phân phối chuẩn tương ứng với độ tin cậy lựa chọn, e là sai số cho phép. Với trường hợp dữ liệu biến động cao nhất $(\mathrm{p}=50 \%)$, với độ tin cậy $95 \%$ và sai số cho phép là $5 \%$ thì ta có cỡ mẫu $n$ được xác định là 384. Do đó, để dự phòng việc mẫu thu về không hợp lệ nên kích cỡ mẫu áp dụng cho nghiên cứu này là 400. Tổng số phiếu tiến hành điều tra là 400 và thực hiện nghiên cứu chính thức là 385 sau khi đã trừ phiếu không hợp lệ (thỏa mãn điều kiện kích thước mẫu cần thiết) được mã hóa và phân tích dữ liệu bằng phần mềm SPSS 25.0 và AMOS 20.0.

Các phương pháp phân tích chủ yếu được sử dụng trong nghiên cứu này là phân tích dưới dạng thống kê mô tả, đánh giá độ tin cậy của thang đo bằng Cronbach's Alpha và EFA, phân tích nhân tố khẳng định CFA và mô hình cấu trúc tuyến tính SEM. Trong phân tích Cronbach's Alpha, để đảm bảo độ tin cậy cao của thang đo thì hệ số Cronbach's Alpha $\geq 0.6$ và hệ số tương quan biến tổng $\geq 0.3$ (Tabachnick \& Fidell, 2013). Hair và cộng sự (1998) đã chỉ ra rằng để đảm bảo cho sự tin cậy và sự phù hợp với thực tiễn của thang đo thì phân tích EFA phải có hệ số tải nhân tố $\geq 0.5$, kiểm định Bartlett's test có ý nghĩa $(\leq 0.05)$, phương sai trích $\geq 50 \%, \mathrm{KMO} \geq 0.5$ và giá trị eigenvalue $>01$. Đối với phân tích $C F A$ và $S E M$, mô hình nghiên cứu phù hợp và tốt với dữ liệu thị trường khi $\mathrm{P}$-value $<0.05 ; \mathrm{CMIN} / \mathrm{df} \leq 2 ; \mathrm{TLI}$ và $\mathrm{CFI} \geq 0.9 ; \mathrm{RMSEA} \leq 0.08$ (Hair et al., 2010).

\section{Kết quả nghiên cứu}

\subsection{Mô tả mẫu nghiên cúu}

Kết quả thống kê mẫu khảo sát cho thấy có 219 khách hàng sử dụng Internet Banking là nữ giới $(56.9 \%)$ và 166 khách hàng là nam giới (43.1\%), với mức thu nhập trung bình của khách hàng là từ 06 triệu đến 15 triệu chiếm đa số (51.2\%), tiếp theo là mức trên 16 triệu đến 25 triệu $(28 \%)$ và dưới 06 triệu $(10.4 \%)$, cuối cùng chiếm tỷ lệ thấp nhất là mức thu nhập từ 25 triệu trở lên $(10.4 \%)$. Khách hàng sử dụng Internet Banking trong mẫu nghiên cứu có nghề nghiệp rất đa dạng, đa phần là nhân viên văn phòng $(67.3 \%)$, tiếp theo là kinh doanh tự do $(16.1 \%)$, giáo viên (7.3\%), sinh viên $(3.5 \%)$, công nhân $(0.8 \%)$ và các nghề nghiệp khác $(5.0 \%)$. Vì vậy, trình độ học vấn của khách hàng cũng khá cao với $79.2 \%$ là có trình độ đại học và $11.2 \%$ trên đại học, còn lại là cao đẳng $(4.7 \%)$, trung cấp $(2.6 \%)$ và PTTH $(2.3 \%)$. Khách hàng cho biết họ đã sử dụng Internet Banking khá lâu, phần lớn là từ 03 đến 06 năm $(59.7 \%)$, tiếp theo là từ 01 đến 03 năm $(16.6 \%)$, từ 07 đến 09 năm $(16.1 \%)$, trên 09 năm $(3.9 \%)$ và cuối cùng là dưới 01 năm $(3,6 \%)$. Đa số khách hàng sử dụng dịch vụ Internet Banking của ngân hàng BIDV (32.2\%), thứ hai là ngân hàng Vietcombank $(25.5 \%)$ và các ngân hàng thương mại khác được khách hàng lựa chọn sử dụng chiếm tỉ lệ dưới $5 \%$, trong đó tần suất sử dụng tập trung ở mức dưới 02 lần/tuần $(36.9 \%)$ và 02 đến 03 lần/tuần $(35.6 \%)$, còn lại là trên 03 lần/tuần. Như vậy, tại Thành phố Hồ Chí Minh, Internet Banking là dịch vụ khá thông dụng và được khách hàng ưu tiên dùng khi có nhu cầu. 


\subsection{Kiểm định độ tin cậy của thang đo bằng Cronbach's Alpha}

Kết quả phân tích hệ số Cronbach's Alpha tổng của các nhân tố đều lớn hơn 0.6 và các hệ số tương quan biến - tổng đều lớn hơn 0.3 nên có thể khẳng định các thang đo trong đề xuất ban đầu đều đều đạt độ tin cậy và được giữ lại để tếp tục đưa vào phân tích nhân tố khám phá EFA.

\section{Bảng 2}

Tổng hợp kết quả kiểm định thang đo

\begin{tabular}{|c|l|c|c|c|c|}
\hline STT & Nhóm biến quan sát & $\begin{array}{c}\text { Số biến } \\
\text { ban đầu }\end{array}$ & $\begin{array}{c}\text { Số biến sau } \\
\text { khi phân } \\
\text { tích }\end{array}$ & $\begin{array}{c}\text { Hệ số tương } \\
\text { quan biến tống } \\
\text { nhỏ nhất }\end{array}$ & $\begin{array}{c}\text { Hệ số } \\
\text { Cronbach's } \\
\text { Alpha }\end{array}$ \\
\hline 1 & Chất lượng thông tin & 7 & 7 & 0.719 & 0.939 \\
\hline 2 & Chất lượng hệ thống & 7 & 7 & 0.615 & 0.912 \\
\hline 3 & Chất lượng dịch vụ & 5 & 5 & 0.803 & 0.944 \\
\hline 4 & Sự tin tưởng & 4 & 4 & 0.661 & 0.843 \\
\hline 5 & Sự hài lòng & 5 & 5 & 0.665 & 0.900 \\
\hline
\end{tabular}

Nguồn: Kết quả phân tích dữ liệu của nhóm tác giả

\subsection{Phân tích nhân tố khám phá EFA}

Tác giả sử dụng phương pháp trích Principal Axis Factoring và phép quay Promax để phân tích nhân tố khám phá EFA. Trong bước này, tác giả thực hiện phân tích EFA 01 lần và kết quả là hệ số $\mathrm{KMO}=0.932$; Kiểm định Bartlett có Sig. $=0.000$ (có ý nghĩa thống kê); các biến quan sát đều có trọng số nhân tố factor loading lớn hơn 0.5 và có 05 nhóm nhân tố được trích tại eigenvalue $1.062(>1)$. Tổng phương sai trích là $72.494 \%>50 \%$. Điều này chứng tỏ $72.494 \%$ biến thiên của dữ liệu được giải thích bởi 05 nhân tố, và 28 biến quan sát gom thành 05 nhân tố. Kết quả phân tích EFA được trình bày cụ thể ở Bảng 3.

\section{Bảng 3}

Kết quả phân tích EFA cho các biến trong mô hình nghiên cứu

\begin{tabular}{|c|c|c|c|c|c|c|}
\hline \multirow{2}{*}{ STT } & \multirow{2}{*}{ Biến quan sát } & \multicolumn{5}{|c|}{ Nhân tố } \\
\cline { 3 - 7 } & CLTT7 & $\mathbf{1}$ & $\mathbf{2}$ & $\mathbf{3}$ & $\mathbf{4}$ & $\mathbf{5}$ \\
\hline 1 & CLTT2 & 0.916 & & & & \\
\hline 2 & CLTT4 & 0.883 & & & & \\
\hline 3 & CLTT5 & 0.878 & & & & \\
\hline 4 & CLTT6 & 0.877 & & & & \\
\hline 5 & CLTT3 & 0.844 & & & & \\
\hline 6 & CLTT1 & 0.841 & & & & \\
\hline 7 & CLHT6 & 0.704 & & & & \\
\hline 8 & CLHT2 & & 0.877 & & & \\
\hline 9 & CLHT5 & & 0.876 & & & \\
\hline 10 & & & & & \\
\hline
\end{tabular}




\begin{tabular}{|c|c|c|c|c|c|c|}
\hline \multirow{2}{*}{ STT } & \multirow{2}{*}{ Biến quan sát } & \multicolumn{5}{|c|}{ Nhân tố } \\
\hline & & 1 & 2 & 3 & 4 & 5 \\
\hline 11 & CLHT3 & & 0.800 & & & \\
\hline 12 & CLHT1 & & 0.757 & & & \\
\hline 13 & CLHT7 & & 0.739 & & & \\
\hline 14 & CLHT4 & & 0.725 & & & \\
\hline 15 & CLDV4 & & & 0.933 & & \\
\hline 16 & CLDV3 & & & 0.913 & & \\
\hline 17 & CLDV5 & & & 0.899 & & \\
\hline 18 & CLDV1 & & & 0.886 & & \\
\hline 19 & CLDV2 & & & 0.859 & & \\
\hline 20 & HL2 & & & & 0.914 & \\
\hline 21 & HL3 & & & & 0.889 & \\
\hline 22 & HL1 & & & & 0.845 & \\
\hline 23 & HL4 & & & & 0.835 & \\
\hline 24 & HL5 & & & & 0.625 & \\
\hline 25 & NT1 & & & & & 0.848 \\
\hline 26 & NT2 & & & & & 0.836 \\
\hline 27 & NT3 & & & & & 0.796 \\
\hline 28 & NT4 & & & & & 0.647 \\
\hline
\end{tabular}

Nguồn: Kết quả phân tích dữ liệu của nhóm tác giả

\subsection{Phân tích nhân tố khẳng định CFA}

Kết quả CFA của mô hình đo lường sau cùng (mô hình tới hạn) cho thấy mô hình có 301 bậc tự do, Chi-square $=538.975$ với $\mathrm{p}=0.000<0.05$; chi-square $/ \mathrm{df}=1.791<2$ các chỉ số $\mathrm{GFI}=$ $0.911 ;$ TLI $=0.964$ và $\mathrm{CFI}=0.972$ lớn hơn $0.9 ; \mathrm{RMSAE}=0.045<0.08$. Các chỉ số trên khẳng định mô hình nghiên cứu là phù hợp với dữ liệu thị trường. Các biến trong mô hình đều có các trọng số chuẩn hóa cao $(>0.5)$, biến CLHT1 $(0.585)$ có trọng số nhỏ nhất và tất cả các biến đều có ý nghĩa thống kê ( $\mathrm{p}$-value $=0.000)$ nên các biến quan sát trong thang đo đều đạt được giá trị hội tụ. Các biến quan sát đều đạt được tính đơn hướng do không có mối tương quan giữa các sai số đo lường. Tất cả các thang đo đều có các giá trị độ tin cậy tổng hợp nằm trong phạm vi được đánh giá là rất tốt, trải dài từ 0.845 đến 0.944 . Bên cạnh đó các giá trị tổng phương sai trích đều lớn hơn 0.5 . Kết quả này cho thấy các thang đo đều đạt độ tin cậy cao. Ngoài ra, các hệ số tương quan của từng cặp khái niệm kèm với sai lệch chuẩn của các thang đo đều khác với 01 ở độ tin cậy $95 \%$, đạt mức ý nghĩa thống kê (tất cả $\mathrm{p}$ đều bằng 0.000 ) nên các biến quan sát dùng để đo lường các khái niệm nghiên cứu đều đạt được giá trị phân biệt.

\subsection{Phân tích mô hình cấu trúc tuyến tính SEM}

Để kiểm định mô hình nghiên cứu, nhóm tác giả đã tiến hành phân tích mô hình cấu trúc tuyến tính (SEM) thông qua phần mềm AMOS cho kết quả cụ thể là: mô hình nghiên cứu có 315 
bậc tự do, Chi-square $=572.322$ với $\mathrm{p}=0.000$; Chi-square $/ \mathrm{df}=1.817<2$; các chỉ số $\mathrm{GFI}=$ $0.905 ; \mathrm{TLI}=0.963, \mathrm{CFI}=0.969$ và $\mathrm{RMSAE}=0.046<0.08$. Do đó, ta có thể kết luận rằng, mô hình nghiên cứu hoàn toàn phù hợp với dữ liệu thị trường. Tuy nhiên, trong mối quan hệ nhân quả giữa các khái niệm với độ tin cậy $95 \%$, cần loại mối quan hệ giữa chất lượng hệ thống với sự hài lòng và chất lượng thông tin với sự hài lòng do có $\mathrm{p}>0.05$ ra khỏi mô hình vì không có ý nghĩa thống kê. Do đó, mô hình có sự cải thiện về thông số Chi-square $=574.317$; Chi-square/df $=1.812<2 ; \mathrm{GFI}=0.904 ; \mathrm{TLI}=0.964 ; \mathrm{CFI}=0.969 ; \mathrm{RMSEA}=0.046$, các chỉ tiêu đánh giá mức độ đều phù hợp với dữ liệu thị trường, có ý nghĩa thống kê vì p-value < 0.05 ở độ tin cậy $95 \%$.

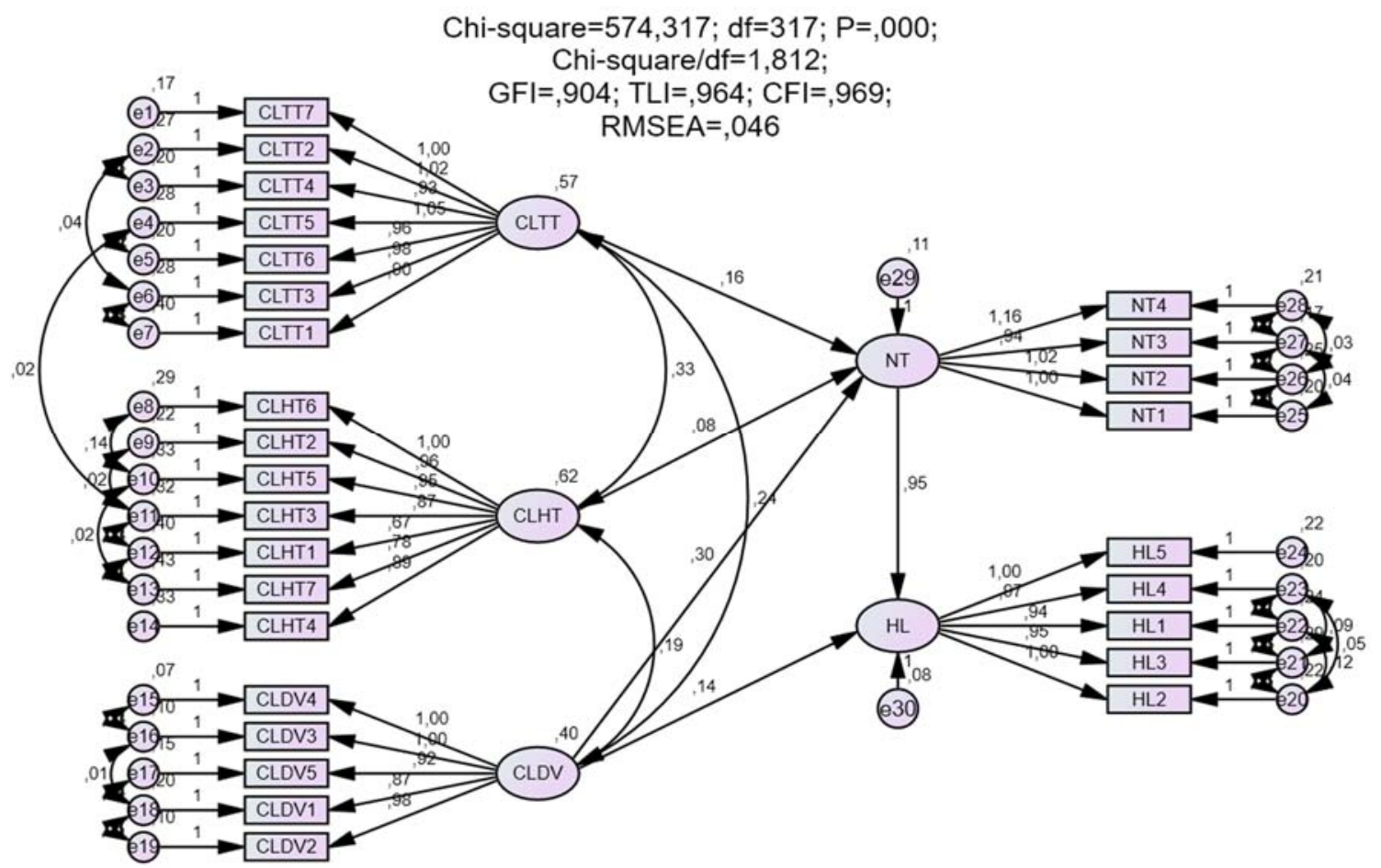

Hình 3. Kết quả SEM chuẩn hóa của mô hình lý thuyết

\subsection{Kiểm định uớc lự̂ng mô hình lý thuyết bằng Boostrap}

Phương pháp Boostrap được áp dụng với số lượng mẫu lặp lại $\mathrm{N}=1,000$. Kết quả phân tích chỉ ra rằng mặc dù có sự xuất hiện của độ lệch nhưng thấp và ổn định (trị tuyệt đối $\mathrm{CR}$ cao nhất là 01 ). Điều này khẳng định ở mức ý nghĩa $5 \%$, ước lượng trong mô hình nghiên cứu này là đáng tin cậy.

\section{Bảng 4}

Kết quả ước lượng Boostrap $(\mathrm{N}=1,000)$

\begin{tabular}{|c|c|c|c|c|c|c|}
\hline Mối quan hệ & SE & SE-SE & Mean & Bias & SE-Bias & CR \\
\hline $\mathrm{NT} \leftarrow \mathrm{CLTT}$ & 0.074 & 0.002 & 0.259 & -0.002 & 0.002 & -1 \\
\hline $\mathrm{NT} \leftarrow \mathrm{CLHT}$ & 0.074 & 0.002 & 0.13 & -0.001 & 0.002 & -0.5 \\
\hline $\mathrm{NT} \leftarrow \mathrm{CLDV}$ & 0.064 & 0.001 & 0.423 & 0 & 0.002 & 0 \\
\hline $\mathrm{HL} \leftarrow \mathrm{CLDV}$ & 0.073 & 0.002 & 0.16 & 0.002 & 0.002 & 1 \\
\hline $\mathrm{HL} \leftarrow \mathrm{NT}$ & 0.081 & 0.002 & 0.759 & -0.001 & 0.003 & -0.333 \\
\hline
\end{tabular}

Nguồn: Kết quả phân tích dữ liệu của nhóm tác giả 


\subsection{Kiểm định giả thuyết của mô hình nghiên cứu}

Kết quả kiểm định giả thuyết của mô hình nghiên cứu ở mức ý nghĩa $5 \%$ trong Bảng 5 cho thấy các nhân tố gồm chất lượng thông tin, chất lượng dịch vụ và chất lượng hệ thống đều có ảnh hưởng thuận chiều đến sự tin tưởng của khách hàng sử dụng Internet Banking. Trong đó, Chất Lượng Dịch Vụ (CLDV) có tác động mạnh nhất đến sự tin tưởng $(\mathrm{NT})$ của khách hàng; tiếp đến là Chất Lượng Thông Tin (CLTT) và tác động yếu nhất là Chất Lượng Dịch Vụ (CLDV). Sự Hài Lòng của khách hàng (HL) chịu ảnh hưởng cùng chiều bởi sự tin tưởng của khách hàng và chất lượng dịch vụ. Tuy nhiên, chất lượng hệ thống và chất lượng thông tin không ảnh hưởng đến sự hài lòng của khách hàng đối với Internet Banking.

\section{Bảng 5}

Kết quả kiểm định các giả thuyết nghiên cứu

\begin{tabular}{|l|c|c|c|c|}
\hline \multicolumn{1}{|c|}{ Mối quan hệ } & Giả thuyết & $\begin{array}{c}\text { Hệ số hồi quy } \\
\text { chuẩn hóa }\end{array}$ & Giá trị P & Kết quả \\
\hline $\mathrm{CLTT} \rightarrow \mathrm{HL}$ & $\mathrm{H} 1 \mathrm{a}$ & -0.003 & 0.964 & Bác bỏ \\
\hline $\mathrm{CLTT} \rightarrow \mathrm{NT}$ & $\mathrm{H} 1 \mathrm{~b}$ & 0.26 & 0.000 & Chấp nhận \\
\hline $\mathrm{CLHT} \rightarrow \mathrm{HL}$ & $\mathrm{H} 2 \mathrm{a}$ & -0.051 & 0.313 & Bác bỏ \\
\hline $\mathrm{CLHT} \rightarrow \mathrm{NT}$ & $\mathrm{H} 2 \mathrm{~b}$ & 0.131 & 0.031 & Chấp nhận \\
\hline $\mathrm{CLDV} \rightarrow \mathrm{HL}$ & $\mathrm{H} 3 \mathrm{a}$ & 0.158 & 0.018 & Chấp nhận \\
\hline $\mathrm{CLDV} \rightarrow \mathrm{NT}$ & $\mathrm{H} 3 \mathrm{~b}$ & 0.423 & 0.000 & Chấp nhận \\
\hline $\mathrm{NT} \rightarrow \mathrm{HL}$ & $\mathrm{H} 4$ & 0.76 & 0.000 & Chấp nhận \\
\hline
\end{tabular}

Nguồn: Kết quả phân tích dữ liệu của nhóm tác giả

\section{Kết luận và khuyến nghị}

Dựa trên nền tảng của mô hình ISS của Delone và Mclean (2003) và kết quả của các bài báo khoa học đã được công bố, nhóm tác giả này đã xây dựng mô hình nghiên cứu về các nhân tố ảnh hưởng đến sự hài lòng của khách hàng cá nhân về dịch vụ Internet Banking tại Thành phố Hồ Chí Minh, gồm 05 nhân tố với 28 biến quan sát. Sau khi tiến hành phân tích hệ số tin cậy Cronbach's Alpha, phân tích nhân tố khám phá EFA, phân tích nhân tố khẳng định CFA, mô hình vẫn còn 05 yếu tố, 28 biến quan sát. Kết quả phân tích mô hình cấu trúc tuyến tính SEM lần thứ nhất, các nhân tố chất lượng hệ thống và chất lượng thông tin trong mối quan hệ với sự hài lòng bị loại do không có ý nghĩa thống kê. Kết quả phân tích SEM lần hai cho thấy 02 nhân tố ảnh hưởng thuận chiều đến sự hài lòng, theo thứ tự độ mạnh giảm dần: Sự tin tưởng và chất lượng dịch vụ. Trong đó, sự tin tưởng chịu sự tác động cùng chiều của 03 nhân tố, sắp xếp theo thứ tự độ mạnh giảm dần như sau: Chất lượng dịch vụ, chất lượng thông tin và chất lượng hệ thống.

Dựa vào kết quả trên, các tác giả đề xuất một số khuyến nghị cho các ngân hàng cung cấp cấp dịch vụ Internet Banking nhằm nâng cao sự hài lòng của khách hàng cá nhân khi sử dụng dịch vụ này, cụ thể là: Đối với chất lượng dịch vụ hỗ trợ khách hàng, các ngân hàng thương mại cần đề ra tiêu chuẩn về dịch vụ hỗ trợ khách hàng, đặc biệt là chú trọng khâu giải đáp thắc mắc và khiếu nại phải được giải quyết nhanh chóng và thỏa đáng. Đối với chất lượng thông tin, các ngân hàng thương mại cần có đội ngũ chuyên nghiệp để kiểm soát và quản lý nội dung thông tin hiển thị trên website của mình. Đối với chất lượng hệ thống của dịch vụ Internet Banking, các ngân hàng thương mại cần chú trọng nâng cao chất lượng an ninh bảo mật cho các giao dịch tài 
chính của khách hàng và khắc phục các điểm yếu trong hệ thống để tránh các hacker tấn công; nâng cao cấu hình của máy chủ của hệ thống Internet Banking để cải thiện tốc độ truy cập giao dịch của dịch vụ; đồng thời cải thiện chất lượng website theo hướng dễ sử dụng cho khách hàng.

\section{LÒ̀I CÁM ƠN}

Nhóm tác giả chân thành cảm ơn Trường Đại học Nông Lâm Thành phố Hồ Chí Minh đã tài trợ kinh phí cho nghiên cứu này.

\section{Tài liệu tham khảo}

Aladwani, A. M., \& Palvia, P. C. (2002). Developing and validating an instrument for measuring user-perceived web quality. Information \& Management, 39(6), 467-476.

Asfour, H. K., \& Haddad, S. I. (2014). The impact of mobile banking on enhancing customers' esatisfaction: An empirical study on commercial banks in Jordan. International Business Research, 7(10), 145-169.

Bailey, J. E., \& Pearson, S. W. (1983). Development of a tool for measuring and analyzing computer user satisfaction. Management science, 29(5), 530-545.

Chang, W. L., Chang, H. C., \& Chang, T. M. (2013). A system dynamics approach to e-service recovery and e-trust. Journal of Information Management, 20(2), 193-218.

Chính phủ Việt Nam. (2016). Đề án Phát triển thanh toán không dùng tiền mặt tại Việt Nam giai doan 2016 - 2020 [Project on developing non-cash payment in Vietnam for the 2016 2020]. Retrieved July 20, 2020, from http://vanban.chinhphu.vn/portal/page/ portal/chinhphu/hethongvanban?class_id=2\&_page=1\&mode=detail\&document_id=187896

Cochran, W. G. (1977). Simple random sampling: Sampling techniques (3rd ed.). Hoboken, NJ: John Wiley \& Sons.

Delone, W. H., \& Mclean, E. R (1992). Information systems success: The quest for the dependent variable. Information Systems Research, 3(1), 60-95.

Delone, W. H., \& Mclean, E. R. (2003). The Delone and Mclean model of information systems success: A ten-year update. Journal of Management Information Systems, 19(4), 9-30.

Do, A. T. N. (2016). Các nhân tố ảnh hưởng đến việc sủ dụng Internet Banking của khách hàng ở các ngân hàng thưong mại Việt Nam [Determinants affecting the use of Internet Banking service by customers in Vietnamese commercial banks] (Doctoral dissertation). National Economics University, Hanoi, Vietnam.

Doll, W. J., \& Torkzadeh, G. (1988). The measurement of end-user computing satisfaction. MIS Quarterly, 12(2), 258-274.

Gable, G. G., Sedera, D., \& Chan, T. (2008). Re-conceptualizing information system success: The IS-impact measurement model. Journal of the Association for Information Systems, 9(7), 377-408.

Ghane, S., Fathian, M., \& Gholamian, M. R. (2011). Full relationship among e-satisfaction, etrust, e-service quality, and e-loyalty: The case of iran e-banking. Journal of Theoretical and Applied Information Technology, 33(1), 1-6. 
Hair, J. F., Anderson, R. E., Babin, B. J., \& Black, W. C. (2010). Multivariate data analysis (7th ed.). New York, NY: Prentice Hall.

Hair, J. F., Black, W. C., Babin, B. J., Anderson, R. E., \& Tatham, R. L. (1998). Multivariate data analysis (5th ed.). Upper Saddle River, NJ: Prentice Hall.

Hoang Liem (2019). Thúc đẩy thanh toán không dùng tiền mặt [Promoting non-cash payments]. Retrieved July 20, 2020, from https://nhandan.com.vn/thong-tin-kinh-te/thuc-day-thanhtoan-khong-dung-tien-mat-360657/

Hsu, S. H. (2008). Developing an index for online customer satisfaction: Adoptation of American customer satisfaction index. Expert Systems with Application, 34(4), 3033-3042.

Hsu, Y. I., \& Nguyen, T. M. (2016). Service quality, customer satisfaction, and customer loyalty of Internet Banking in Vietnam. International Review of Management and Business Research, 5(4), 1485-1496.

Iivari, J. (2005). An empirical test of the Delone-Mclean model of information system success. The DATA BASE for Advances in Information Systems, 26(2), 8-27.

Koo, C., Wati, Y., \& Chung, N. (2013). A study of mobile and internet banking service: Applying for IS success model. Asia Pacific Journal of Information Systems, 23(1), 65-86.

Le, N. M. (2019). Kiểm soát các hình thức thanh toán trong khu vực kinh tế phi chính thức ở Việt Nam [Controlling forms of payment in Vietnam's informal economy]. Retrieved July 20, 2020, from https://tapchitaichinh.vn/ngan-hang/kiem-soat-cac-hinh-thuc-thanh-toan-trongkhu-vuc-kinh-te-phi-chinh-thuc-o-viet-nam-312063.html

Lee, K. C., \& Chung, N. (2009). Understanding factors affecting trust in and satisfaction with mobile banking in Korea: A modified Delone and Mclean's model perspective. Interacting with Computers, 21(5/6), 385-392.

Liao, Z., \& Cheung, M. T. (2002). Internet-based e-banking and consumer attitudes: An empirical study. Information \& Management, 39(4), 283-295.

Liu, C., \& Arnett, K. P. (2000). Exploring the factors associated with website success in the context of electronic commerce. Information \& Management, 38(1), 23-33.

Liu, X., He, M., Gao, F., \& Xie, P. (2008). An empirical study of online shopping customer satisfaction in China: A holistic perspective. International Journal of Retail \& Distribution Management, 36(11), 919-940.

Madu, C. N., \& Madu, A. A. (2002). Dimensionsofe-quality. International Journal of Quality \& Reliability Management, 19(3), 246-258.

Morgan, R. M., \& Hunt, S. D. (1994). The commitmenttrust theory of relationship marketing. Journal of Marketing, 58, 20-38.

Mukherjee, A., \& Nath, P. (2003). A model of trust in online relathionship banking. International Journal of Bank Marketing, 21(1), 5-15.

Musiime, A., \& Ramadhan, M. (2011). Internet banking, consumer adoption and customer satisfaction. African. Journal of Marketing Management, 3(10), 261-269.

Ngân hàng Nhà nước Việt Nam. (2016). Thông tu số 35/2016/TT-NHNN ngày 29 /12/2016, Quy định về an toàn, bảo mật cho việc cung cấp dịch vụ ngân hàng trên Internet [Circular No. 35/2016/TT-NHNN dated december 29, 2016, on safety and confidentiality over provision of 
banking services on the Internet]. Retrieved July 21, 2020, from https://thuvienphapluat.vn/van-ban/Tien-te-Ngan-hang/Thong-tu-35-2016-TT-NHNN-antoan-bao-mat-cho-viec-cung-cap-dich-vu-ngan-hang-tren-Internet-333481.aspx

Nimako, S. G., Gyamfi, N. K., \& Wandaogou, A. M. M. (2013). Customer satisfaction with Internet Banking service quality in the Ghanaian banking industry. International Journal of Scientific \& Technology Research, 2(7), 165-175.

Sedera, D., Gable, G., \& Chan, T. (2004). A factor and structural equation analysis of the enterprise systems success measurement model. Proceedings of the 10th Americas conference on information systems (pp. 676-682). Atlanta, GA: Association for Information Systems.

Seyal, A. H., \& Rahim, M. M. (2011). Customer satisfaction with internet banking in Brunei Darussalam: Evaluating the role of demographic factors. E-Service Journal: A Journal of Electronic Services in the Public and Private Sectors, 7(3), 47-68.

Tabachnick, B. G., \& Fidell, L. S. (2013). Using multivariate statistics (6th edition). Boston, MA: Pearson.

Tran, M. T., \& Nguyen, K. M. (2011). Các nhân tố ảnh hưởng đến sự hài lòng về chất lượng dịch vụ Internet Banking của khách hàng cá nhân [Factors affecting the individual customer's satisfaction at Internet banking service quality]. Tap chí Khoa hoc, 5(23),16-29.

VA. (2019). Hệ sinh thái số sẽ thúc đẩy thanh toán không dùng tiền mặt [Digital ecosystem will promote non-cash payment]. Retrieved July 21, 2020, from http://tapchinganhang.gov.vn/hesinh-thai-so-se-thuc-day-thanh-toan-khong-dung-tien-mat.htm

Vuong, Q. D. H., \& Nguyen, Q. M. (2016). Vai trò của Internet Banking và bài học kinh nghiệm cho ngân hàng thương mại Việt Nam [The role of Internet Banking and lessons from experience for commercial banks in Vietnam]. Tạp chí Khoa học- Đại học Văn Hiến, 10, 42-49.

We Are Social., \& Hootsuite. (2020). Báo cáo Digital 2020 trên thế giới và Việt Nam [Digital 2020 report: Global and Vietnam]. Retrieved July 20, 2020, from https://advertisingvietnam.com/downloadbao-cao-tinh-hinh-digital-nam-2020-tai-thi-truongviet-nam

Widiatmika, I. G. N. A., \& Subawa, N. S. (2017). Effect of service quality and recovery service quality mobile banking service to e-trust, e-satisfaction and e-loyalty mobile banking users of local bank customer in Bali. Imperial Journal of Interdisciplinary Research (IJIR), 3(3), 1252-1260.

Yousafzai, S. Y., Pallister, J. G., \& Foxall, G. R. (2003). A proposed model of e-trust for electronic banking. Technovation, 23(11), 847-860.

Zviran, M., Glezer, C., \& Avni, I. (2006). User satisfaction from commercial web sites: The effect of design and use. Information \& Management, 43(2), 157-178.

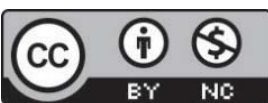

Creative Commons Attribution-NonCommercial 4.0 International License. 